\title{
Asociación espacial entre fragmentación socioeconómica y ambiental en la ciudad de Mar del Plata, Argentina
}

Juan P. Celemín. Consejo Nacional de Investigaciones Científicas y Técnicas (CONICET), Centro de Investigaciones Geográficas, Universidad Nacional del Centro de la Provincia de Buenos Aires, Buenos Aires, Argentina.

RESUMEN | ¿Existe relación entre el nivel socioeconómico de las personas y la calidad ambiental del entorno en que viven? Para responder a tal interrogante se recurrió a la autocorrelación espacial, de manera de asociar un índice socioeconómico con otro de calidad ambiental a los radios censales que conforman la localidad de Mar del Plata (Argentina), lo que permitió obtener una respuesta positiva a la pregunta. Es decir, aquellos que poseen recursos económicos tienen la capacidad de agenciarse un ambiente sano en el cual desarrollarse. La integración de las variables de ambos índices posibilitó realizar otro análisis de tipo cluster, que registró una configuración espacial similar a la obtenida con la autocorrelación espacial. Así quedó plasmada la necesidad de mejorar las condiciones ambientales en las que se encuentran los sectores menos favorecidos, dado que, además del contexto económico, un ambiente sano es uno de los factores necesarios para una buena calidad de vida.

PALABRAS ClAVE | fragmentación urbana, segregación, calidad de vida, calidad ambiental, indicadores ambientales.

ABSTRACT | Is there a relationship between peoples' socioeconomic level and the quality of the environment in which they live? To answer this question, spatial autocorrelation was used to associate a social and economic index with an environmental one, using the census tracts of Mar del Plata (Argentina). This demonstrated a positive answer to the question, i.e. those who possess economic resources have the capacity to live in a healthy environment. The integration of the variables of these two indices in a cluster analysis showed a spatial configuration similar to that obtained with the spatial autocorrelation. This reflected the need to improve environmental conditions in those less favored sectors as, in addition to the economic context, a healthy environment is one of the factors necessary for good quality of life.

KEY WORDS | urban fragmentation, segregation, quality of life, environmental quality, environmental indicators. 


\section{Introducción}

La globalización se caracteriza por la excesiva concentración de los medios de producción y comercialización, así como de los medios financieros y de servicios en el plano mundial y nacional en un contexto de apertura indiscriminada de los mercados. La mundialización económica promueve la integración a la vez que la exclusión cercena la soberanía nacional y aumenta la autonomía del mercado. Este proceso se impone al mundo no solo a través de la internacionalización de la economía, sino también de la cultura y del consumo, entre otros aspectos, un proceso cuya finalidad es la homogeneización de la sociedad y del territorio. Ello ocurre, sin embargo, a costa de una gran cantidad de fracturas, por lo que se considera que este modelo va acompañado de otro, denominado fragmentación (Uribe Ortega, 1998).

Este esquema global, además de cambiar el tejido económico y social y el medioambiente de las grandes áreas urbanas, también reforma su estructura espacial (Sachs-Jeantet, 1994, p. 7). Consecuentemente, su marca queda registrada en la territorialidad de las ciudades al acentuar sus disparidades internas, ya que algunos sectores se ven favorecidos por ese proceso de internacionalización de la economía, mientras que otros, la mayoría, quedan marginalizados.

La supuesta modernización es la fuerza motriz que anima la vida de relaciones de países y lugares, merced a la incorporación diferencial y selectiva, por parte de cada territorio, de los datos centrales de cada período histórico. Así, a cada totalidad le corresponde una modernidad respectiva, provista de nuevos significados, contenidos, jerarquías y valores. De este modo, la modernización no es lineal ni unívoca. Una etapa no sucede mecánicamente a la otra, ni una es el producto inequívoco de la anterior. No existen situaciones geográficas que puedan ser explicadas únicamente mediante una referencia a su pasado. En efecto, la naturaleza central del problema no reside en áreas modernizadas diferencialmente, sino, por el contrario, en la coexistencia espacio-temporal de diversas modernidades, esto es, diversas formas de modernización, articuladas entre sí por y en el proceso de totalización. Cada modernidad es una totalidad concreta, una forma de ser del mundo, con sus posibilidades y sus limitaciones, que se objetiva, se funcionaliza y se plasma en uno o más puntos del espacio. El proceso de modernización, comandado a cada momento de la historia y en cada lugar por las clases dominantes — capitales hegemónicos, el propio Estado-, lejos de suprimir las desigualdades preexistentes, las exacerba. Y así, durante el devenir espaciotemporal de los fenómenos sociales, engendra nuevos mecanismos de diferenciación y fragmentación de la sociedad y el territorio, merced a la funcionalización de nuevos sistemas, objetos, acciones y normas. En este contexto, la calidad de vida es tributaria de ese proceso de modernización, pues deviene medio y resultado de las nuevas racionalidades y usos del territorio (Gómez Lende, 2005, p. 243).

El estudio de la fragmentación urbana no ha dejado de provocar desacuerdos, tanto en la definición y límites del término, como en sus métodos y técnicas de medición (González Arellano, 2006). Esto se debe a que su análisis no es propiedad 
exclusiva de una disciplina específica, de manera que cada una la entiende y examina de acuerdo con sus lenguajes y metodologías A su vez, la mayoría de los conceptos asociados a la temática (vulnerabilidad social y segregación, por nombrar algunos) también son multidimensionales, están en permanente discusión y su delimitación continúa abierta. No obstante, se reconoce que la fragmentación repercute en la configuración urbana, y en particular en la de las ciudades de América Latina, según las modalidades que adquiere el impacto del capitalismo internacional en las diferentes culturas de los países dependientes de la región.

La diferenciación espacial ha existido siempre; sin embargo, en la actualidad se observa con mayor nitidez y amplitud en los espacios de ciudades en desarrollo. Particularmente se presenta una clara distinción entre los que tienen mucho y los que no tienen; es decir, se exhibe un espacio más fragmentado y segregado. En este marco, Alvarado Rosas, Medrano \& Lozano (2008) y Janoschka (2002) mencionan que existe una gran fragmentación de los usos del suelo como nuevas formas de la expresión espacial de las ciudades de América Latina a finales del siglo veinte y principios del veintiuno. Asimismo, es común que el suelo público se vea cada vez más afectado por el fenómeno de la privatización, proceso que ha trascendido varios espacios de la economía de las ciudades. Ello es especialmente notorio en las áreas donde se han promovido fuertemente las inversiones inmobiliarias, enfocadas a los fraccionamientos cerrados de alto nivel, que prácticamente construyen pequeñas ciudades dentro de otra gran ciudad. A la vez crecen los espacios de pobreza, zonas marginadas destinadas a la población de bajos ingresos, que se encuentran aislados por grupos de población de nivel socioeconómico mayor. Esta característica también es mencionada en los trabajos de Meyer y Bähr (2001) y De Mattos e Hidalgo (2007).

Por su parte, Sabatini, Cáceres y Cerda (2001) discuten teóricamente la relación entre desigualdad social y segregación espacial, rechazando el enfoque usual que ve la segunda como un mero reflejo en el espacio de la primera, mientras que Borsdorf (2003) sostiene la necesidad de recurrir a nuevos modelos para el análisis urbano, que deben ser generalizaciones de la realidad fragmentada de la ciudad latinoamericana actual.

La gran mayoría de los trabajos que analizan la fragmentación lo hacen, como es de esperar, desde una perspectiva socioeconómica y aplicando técnicas estadísticas tradicionales (correlación entre variables, por ejemplo). Consiguientemente, son escasas las publicaciones que contemplan el uso explícito de procedimientos de análisis espacial para el estudio de la segregación urbana y también son pocas las producciones que vinculan el ambiente urbano como un elemento más que puede ser considerado al momento de estudiar la fragmentación. Entre las primeras se puede mencionar los recientes trabajos de Linares $(2008,2010)$ sobre ciudades intermedias argentinas; a Groisman y Suárez (2006) con el estudio de la segregación residencial en Buenos Aires; Kaztman y Retamoso (2007) con el análisis de la segregación educativa en Montevideo; y Martori, Hoberg y Surinach (2006) para Barcelona. Todos ellos recurren a la autocorrelación espacial — por ejemplo, los habituales coeficientes de correlación - como medida para mensurar la fragmen- 
tación en sus áreas de estudio, dejando de lado técnicas cuantitativas no-espaciales. Más limitada es la producción que analiza el vínculo entre la distribución espacial de los servicios ambientales y el contexto socioeconómico urbano a partir del uso de estas técnicas. Una variable ambiental de fácil relevamiento, que ha sido utilizada en diferentes trabajos para relacionarla espacialmente, de manera más o menos explícita, con la segregación socioeconómica, es la distribución de la vegetación urbana. Así, Escobedo et al. (2006) establecen su vínculo con los estratos socioeconómicos de las comunas de Santiago de Chile; Pedlowski, Carneiro Dasilva, Adell y Heynen (2006) la asocian con la inequidad ambiental de Campos dos Goytacazes (Brasil); y Jensen (2004) y Mennis (2006) estudian su vínculo con la calidad de vida para localidades estadounidenses a partir de la elaboración de un índice "verde", que es contrastado con variables socioeconómicas. Otro trabajo interesante es el de Santana Rodríguez, Escobar Jaramillo y Capote (2010) que combinan variables socioeconómicas y ambientales a partir de la integración de información censal y de imágenes satelitales de la ciudad de Cali (Colombia), para luego realizar un análisis factorial. Entre los índices ambientales más recientes se puede mencionar el de Celemín y Velázquez (2011), que elaboran un índice a escala departamental para la provincia de Buenos Aires constituido no solo por las habituales variables físico-naturales, sino también por aquellas referidas al ambiente urbano construido.

Queda, entonces, plasmada la necesidad de incorporar de manera explícita la dimensión ambiental en el estudio de la polarización; en otras palabras, demostrar en el espacio urbano que quienes se encuentran en un mejor contexto socioeconómico son los mismos que pueden acceder a una calidad ambiental aceptable. Consiguientemente, cuando el acceso a los bienes y servicios ambientales y la posibilidad de evitar efectos negativos están determinados esencialmente por el nivel de ingreso, los costos recaen invariablemente sobre las franjas más débiles y pobres de la población. Así, surge el objetivo del presente trabajo: determinar territorialmente el grado de asociación entre ambas dimensiones en la ciudad de Mar del Plata, una localidad de 600.000 habitantes que se encuentra ubicada sobre el océano Atlántico, en la zona sudeste de la provincia de Buenos Aires de la República Argentina, y que es cabecera del Partido de General Pueyrredón (Figura 1). Para ello se busca relacionar espacialmente, por medio de la autocorrelación espacial, un índice socioeconómico con otro de índole ambiental que no solo contempla la vegetación, tal como lo hicieron los trabajos mencionados con anterioridad.

Un ejemplo claro de vinculación entre ambiente e ingreso se produce con lo que se conoce como "tragedia de los comunes", que ocurre cuando los servicios ambientales supuestamente gratuitos - sean ellos recursos como el aire, el agua o los espacios verdes, entre otros- y que son de carácter público, son apropiados para usos privados por los sectores más acomodados. La consecuencia es un tipo de ciudad que, en rasgos generales, aparece fragmentada entre un sector social de la población con acceso al consumo y los servicios, y otro sumido en la pobreza y a pocos pasos de la exclusión social. En otras palabras, la inequidad social se refleja en la producción y mantenimiento de la ciudad. Existe una ciudad para los que la pueden pagar, 
con todos los servicios que los avances técnicos permiten, con una sana situación de legalidad y, en la mayoría de nuestras ciudades, con un medioambiente circundante con pocos signos de deterioro. La otra ciudad es inundable, sin servicios ni equipamientos, sin una seguridad en la situación legal, con un entorno ambiental deteriorado. La polarización cada vez mayor que se da en la sociedad se refleja, así, en la apropiación y construcción del ambiente urbano (Clichevsky, 2002, p. 12).

La contribución empírica que puede realizarse desde el análisis de los datos georreferenciados consiste en reconocer que la localización en el espacio tiene mucha importancia en las condiciones de vida de las personas. En tal sentido, las diferencias territoriales son un reflejo de las inequidades de la población dentro de una sociedad definida temporal y espacialmente.

FIGURA 1 | Ubicación de la ciudad de Mar del Plata, en la provincia de Buenos Aires, Partido de General Pueyrredón, Argentina

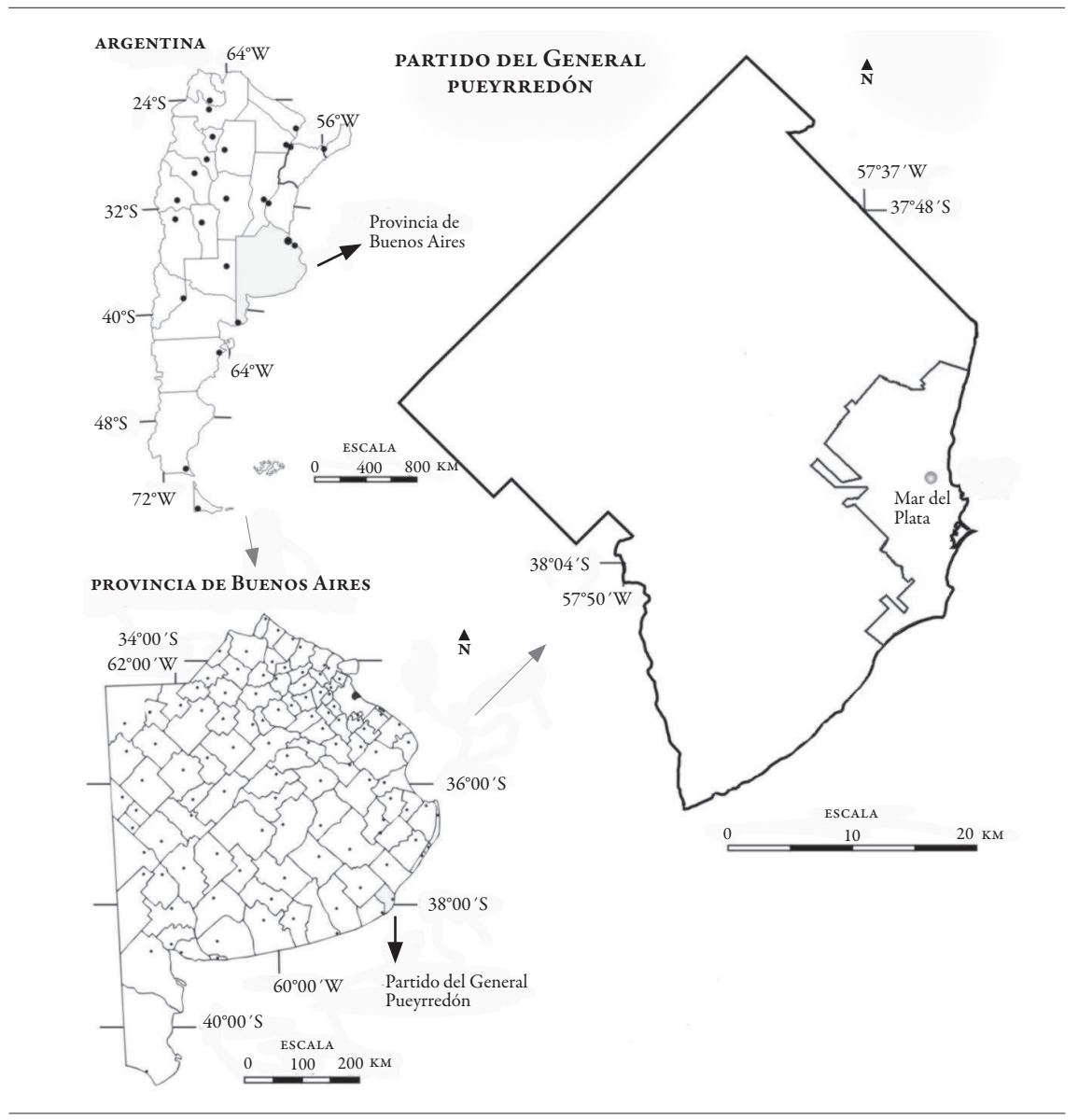

FUENTE ELABORACión PROPIA. 
El proceso contemporáneo de fragmentación social y territorial de las condiciones de vida de la población se manifiesta, entonces, como un nuevo mecanismo de exclusión social y segregación espacial, que transforma sustancialmente la dinámica y la configuración de un diverso mosaico de subespacios. Un abordaje riguroso y sistemático de la naturaleza de esta situación resulta fundamental para la investigación urbana. El proceso histórico genera así la necesidad continua y sistemática de adaptar la construcción metodológica de índices a las nuevas condiciones socioespaciales inherentes al período contemporáneo (Cepeda Marinelli, Gómez Lende \& Velázquez, 2004) y, para ello, es fundamental recurrir a las nuevas tecnologías y procedimientos que permiten un análisis detallado de las configuraciones sociales y ambientales en el espacio urbano. Con tal fin se emplean medidas incluidas en el estudio de la autocorrelación espacial, que se vislumbra como un fundamento propio del análisis de datos georreferenciados (Lucero \& Celemín, 2008), y cuyas técnicas vienen evolucionando debido al particular interés por reconocer los fenómenos espaciales con la mayor profundidad posible. A dicho estudio se suma el análisis cluster. El primero sirve para establecer si los índices propuestos están correlacionados espacialmente, mientras que el segundo procedimiento los integra, para luego poder cartografiar los agrupamientos de las variables.

\section{Metodología}

Para realizar el análisis de los datos georreferenciados es fundamental recurrir a las herramientas informáticas desarrolladas en los últimos años en el ámbito del análisis de la información espacial, resaltando la potencialidad que tienen para estudiar la distribución de índices y variables en el espacio, mensurando y localizando las diferentes configuraciones territoriales como forma de diagnóstico ante los problemas urgentes y emergentes. Para obtener un alto grado de detalle, es muy importante recurrir a la mínima unidad espacial disponible; en este caso, el radio censal, compuesto por aproximadamente un conjunto de trescientas viviendas. De esta manera se minimiza el efecto conocido como Problema de la Unidad Espacial Modificable, en el cual, en la medida en que las unidades espaciales se agrupan para formar otras de mayor tamaño (de radios a fracciones, de estas a municipios, y de estos a provincias, por ejemplo), las correlaciones entre las variables estudiadas tienden a aumentar debido al promedio de los datos que se realiza al pasar de una escala a otra (Openshaw, 1984). En la localidad de Mar del Plata, dicha escala está compuesta por un total de 739 radios censales.

\section{Elaboración de índices}

El proceso metodológico de construcción de un índice presenta una gran complejidad, dado que deben seleccionarse variables representativas de la situación que se pretende mostrar. Además, su implementación varía según los ámbitos geográficos para los cuales está propuesto y diseñado. Este tipo de agrupación es una forma de organizar mejor la información de acuerdo con la similitud existente entre las 
variables y la finalidad del índice. Tanto en la forma de agruparlas como en su ponderación existe un componente subjetivo, producto de los conocimientos del investigador y de las referencias consultadas.

En muchos casos, la disponibilidad de esa información es inversa a la escala de análisis, dado que gran parte de las variables disponibles para el país en su totalidad no lo están a escala provincial y, a su vez, no lo están para el nivel departamental o para niveles más detallados, como los de fracción o radio censal. Esta suerte de paradoja de la información geográfica implica que la información más interesante esté disponible solamente para escalas poco interesantes, y en la medida en que el nivel de análisis espacial se incrementa, la disponibilidad de información disminuye (Velázquez, 2008, p. 26). Consecuentemente, la pertenencia de las variables a una dimensión determinada no es definitiva, ya que alguna puede pertenecer a más de una. Por lo tanto, en algunos casos hay que recurrir a variables que se aproximan a las que el investigador desearía utilizar.

En el Cuadro 1 se desagrega la información del Índice Socioeconómico (ISE), mientras en el Cuadro 2 se hace lo mismo con el Índice de Calidad Ambiental (ICA), que fueron representados cartográficamente mediante un Sistema de Información Geográfica (SIG). La información proviene mayoritariamente del último Censo Nacional de Población, Vivienda y Hogares $(\mathrm{CNPVyH})$ realizado el año 2001. A la vez, los datos ambientales fueron complementados con aportes provenientes de la Secretaría de Política Ambiental de la Provincia de Buenos Aires y de dependencias de la Municipalidad de General Pueyrredón.

CUADRO 1 | Dimensiones, variables y ponderaciones utilizadas en el Índice Socioeconómico

\begin{tabular}{l|l|c}
\hline \multirow{2}{*}{ DIMENSIÓN } & VARIABLES & PONDERACIÓN \\
\hline \multirow{2}{*}{ EDUCACIÓN } & $\begin{array}{l}\text { Porcentaje de población de } 15 \text { años o más que ya no asiste y con nivel de instrucción } \\
\text { menor a primario completo. }\end{array}$ & 1,25 \\
\cline { 2 - 3 } & $\begin{array}{l}\text { Porcentaje de población de 20 y más años que ya no asiste y con nivel de instrucción } \\
\text { universitario completo. }\end{array}$ & 1,25 \\
\hline \multirow{2}{*}{ SALUD } & Porcentaje de población que posee obra social o cobertura médica asistencial. & 1,50 \\
& Porcentaje de población con provisión de agua por cañería dentro de la vivienda. & 1,50 \\
\hline \multirow{2}{*}{ VIVIENDA } & Porcentaje de población en hogares con baño de uso exclusivo. & 1,50 \\
\hline & Porcentaje de población en viviendas con CALMAT I.* & 1,00 \\
\hline \multirow{2}{*}{ EQUIPAMIENTO } & Porcentaje de población en hogares con hacinamiento (dos y más personas por cuarto). & 1,00 \\
\hline & Porcentaje de hogares con tenencia de computadora con conexión a internet. & 0,50 \\
\hline
\end{tabular}

* CALMAT: Calidad de los materiales de la vivienda. CALMAT I: La vivienda presenta materiales resistentes y sólidos en todos los parámetros (pisos, paredes o techos) e incorpora todos los elementos de aislación y terminación. Véase Municipalidad de La Plata, Estadistica y evaluación de programas especiales. En http://www.estadistica.laplata.gov.ar/paginas/CENSO/3\%20 Viviendas/v3-6.pdf 
CUADRO 2 | Dimensiones, variables y ponderaciones utilizadas en el Índice de Calidad Ambiental

\begin{tabular}{|c|c|c|}
\hline DIMENSIÓN & VARIABLE & PONDERACIÓN \\
\hline \multirow{2}{*}{ USO DEL AGUA } & $\begin{array}{l}\text { Porcentaje de población que reside en hogares con inodoro con descarga de } \\
\text { agua y desagüe a red pública. }\end{array}$ & 2,00 \\
\hline & $\begin{array}{l}\text { Porcentaje de población que reside en viviendas con agua proveniente de la } \\
\text { red pública. }\end{array}$ & 2,00 \\
\hline \multirow{2}{*}{$\begin{array}{l}\text { RECOLECCIÓN DE } \\
\text { RESIDUOS }\end{array}$} & $\begin{array}{l}\text { Porcentaje de población que reside en viviendas con servicio regular de } \\
\text { recolección de residuos. }\end{array}$ & 1,50 \\
\hline & Superficie en metros cuadrados de vacíos urbanos por habitante. & 0,75 \\
\hline \multirow{2}{*}{ CALIDAD DEL AIRE } & $\begin{array}{l}\text { Superficie en metros cuadrados de espacios verdes por habitante (públicos y } \\
\text { privados). }\end{array}$ & 1,00 \\
\hline & $\begin{array}{l}\text { Porcentaje de la superficie del radio censal a menos de } 201 \text { metros de } \\
\text { fábrica(s). }\end{array}$ & 0,75 \\
\hline RIESGO NATURAL & Población por hectárea en condiciones de riesgo de inundabilidad. & 1,00 \\
\hline ACCESIBILIDAD & $\begin{array}{l}\text { Porcentaje de la superficie del radio censal a menos de } 501 \text { metros de } \\
\text { espacio(s) verde(s) público(s) y a menos de } 1001 \text { metros de la costa. }\end{array}$ & 1,00 \\
\hline TOTAL & & 10,00 \\
\hline
\end{tabular}

Los valores para cada variable y unidad espacial fueron estandarizados de acuerdo con las siguientes fórmulas matemáticas y su sentido positivo o negativo.

Variables cuyo incremento implica peor situación relativa:

$$
P E_{I}=\frac{M-x_{i}}{M-m}
$$

Variables cuyo incremento implica mejor situación relativa:

$$
P E_{I}=1 \frac{M-x_{i}}{M-m}
$$

siendo $P E_{i}=$ puntaje estándar de $i$-esimo dato, $x_{i}=$ el dato original por ser estandarizado. 


\section{Autocorrelación espacial}

Los índices de autocorrelación espacial permiten relacionar en forma conjunta la dependencia entre lugares y valores de variables o atributos que interesan. A la vez, resultan muy adecuados para observar la configuración espacial fragmentada propia de nuestros tiempos.

Definida de una manera estadística, se considera autocorrelación espacial de primer orden aquella influencia que ejerce un punto $i$ sobre sus vecinos contiguos, mientras que hay autocorrelación espacial de un orden mayor cuando también se consideran los vecinos a las observaciones $j$. También se pueden considerar los vecinos que se localicen a cierta distancia $d$ de la observación $i$.

Según lo expuesto con anterioridad, queda claro que la intención del análisis es medir la asociación entre lugares distintos según atributo o variable de interés, pero teniendo en cuenta cuál es la posición relativa de los lugares involucrados.

$\mathrm{Si}$ se intenta medir la correlación que una misma variable tiene en diferentes unidades espaciales contiguas en una perspectiva horizontal, se puede registrar una de las siguientes tres posibilidades:

- Autocorrelación espacial positiva: las unidades espaciales vecinas presentan valores próximos. Indica una tendencia al agrupamiento de las unidades espaciales.

- Autocorrelación espacial negativa: las unidades espaciales vecinas presentan valores muy disímiles. Indica una tendencia a la dispersión de las unidades espaciales.

- Sin autocorrelación: no ocurre ninguna de las dos situaciones anteriores. Por lo tanto, los valores de las unidades espaciales vecinas presentan valores producidos en forma aleatoria.

Con base en el estadístico general se crearon diferentes índices para medir la autocorrelación espacial. El primero, establecido por Moran en 1950 y perfeccionado en el transcurso de los años, es análogo al coeficiente de correlación usual entre dos variables. A pesar de su antigüedad no fue muy utilizado, debido a que el cómputo manual era sumamente laborioso; fue necesario contar con programas informáticos para poder realizarlo. Es por ello que recién en la década de los noventa fue rescatado del olvido y comenzó a utilizarse en trabajos de investigación de manera consistente.

El programa utilizado para aplicar la autocorrelación espacial es el GeoDa, elaborado por la University of Illinois, Urbana-Champaign, de carácter gratuito, que se enmarca en lo que se conoce como un software para el Análisis Exploratorio de Datos Espaciales (ESDA, por su sigla en inglés). Este programa busca crear una interfaz intuitiva y fácil de usar para estudiar la información geográfica, que aliente la exploración y permita a los usuarios descubrir patrones y anomalías en los datos que de otro modo no serían aparentes. Como tal, las pruebas de su éxito parecen estar más asociadas con los programas de análisis estadístico tradicionales que con 
los SIG, más reconocidos por su complejidad y por el largo aprendizaje necesario para poder manejarlos.

\section{Análisis cluster}

El segundo procedimiento es más conocido. Se lo denomina análisis cluster y es utilizado para obtener grupos homogéneos de unidades espaciales y maximizar la heterogeneidad entre los grupos formados. Por lo tanto, desde el análisis espacial se le podría incluir el concepto de regionalización, dado que los objetos por clasificar son unidades espaciales que, una vez unidas, generan regiones en el espacio (Buzai, 2003, p. 186). Asimismo, la excelente capacidad de clasificación que este procedimiento presenta permite identificar claramente las relaciones multivariadas que difícilmente son accesibles a partir del análisis de las unidades espaciales individuales. En este estudio se empleó un cluster jerárquico ascendente, considerando como medida de distancia la euclídea al cuadrado, y el método de mínima variación intragrupo (método de Ward). El mismo se diferencia de otros por realizar en cada etapa todas las uniones posibles, eligiendo aquella que genera una menor variación en el interior de los grupos. En este caso se recurrió al Philcarto 5.xx para el análisis, un software francés que, como el anterior, también es gratuito y puede ser considerado como un ESDA.

\section{Resultados}

\section{Distribución espacial de los índices}

El resultado final del Índice Socioeconómico (ISE) para la localidad de Mar del Plata muestra una distribución espacial con patrones bien definidos (Figura 2). Se establecieron cuatro categorías que permiten observar cómo, desde el centro urbano hacia la periferia, la disminución del índice se hace evidente, la fragmentación socioeconómica es más nítida y, por lo tanto, la representación territorial se torna más patente. Se puede distinguir que parte del centro no está en el rango de valores máximos. Esto podría estar reflejando cierto deterioro en un contexto de envejecimiento edilicio, en una zona sin margen para las nuevas construcciones, que propicia una búsqueda de nuevos lugares por parte de determinados grupos sociales, en sitios puntuales en zonas aledañas o directamente en la periferia. En este último caso predominaría el interés por los espacios verdes y "alejarse del ruido del centro". La situación podría ser potenciada por el surgimiento de nuevas áreas comerciales, de carácter más exclusivo.

El Índice de Calidad Ambiental (ICA) para la ciudad de Mar del Plata tiene una distribución más fragmentada que el anterior (Figura 3). En general, se observa una preponderancia de los valores altos y medios altos, a la vez que, como en el caso del ISE, la periferia registra las puntuaciones más bajas de toda la localidad. 
FIGURA 2 | Índice Socioeconómico para la ciudad de Mar del Plata

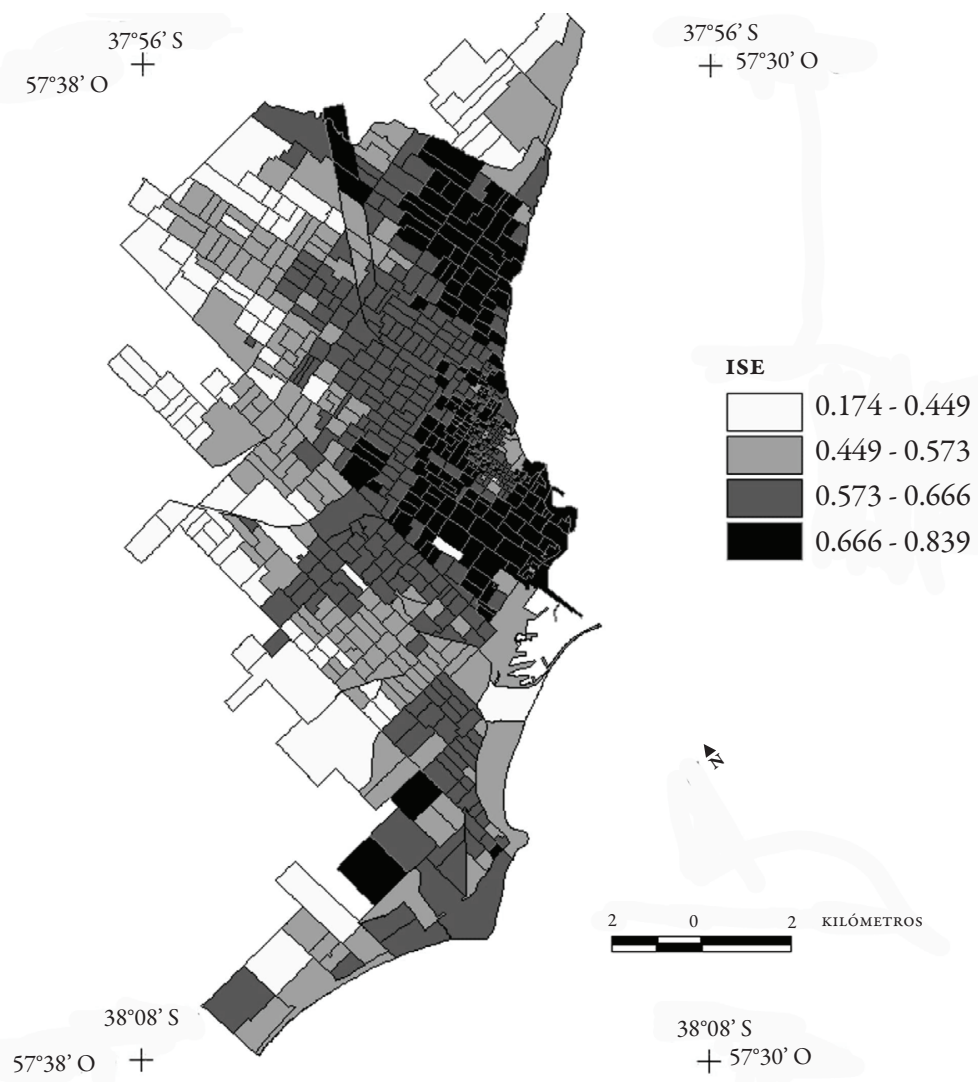

fuente Elaboración propia con base en Cartografía del Censo 2001

Autocorrelación bivariada del Índice Socioeconómico con el Índice de Calidad Ambiental

La comparación de ambos índices se realizó mediante un análisis de autocorrelación bivariado en el cual el ISE se ubica en el eje de las $x$ y el ICVA en el eje de las $y$. El resultado refleja un valor medio para $I$ de Moran de 0,5126 que se diferencia del valor esperado para ese índice si la configuración espacial hubiese sido aleatoria, $E(I)=-0,0014$ con un $p$-valor de 0,0010 , que implica una muy baja probabilidad de error. La información desagregada por cuadrante está en el Cuadro 3. 
FIgura 3 | Índice de Calidad Ambiental para la ciudad de Mar del Plata

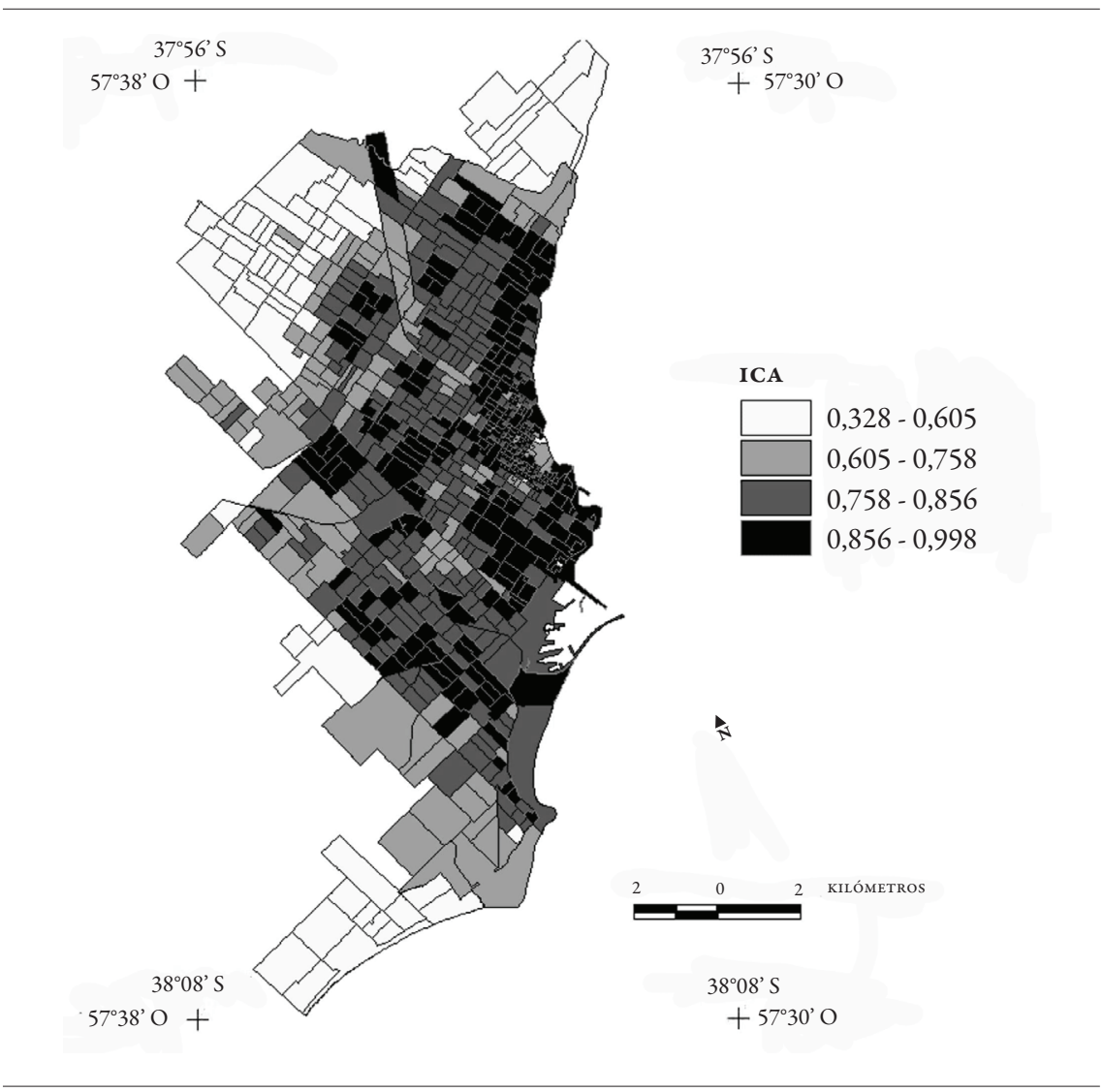

FUENTE Elaboración PRopia CON BASE en CARTografía del Censo 2001.

CUADRO 3 | Autocorrelación espacial bivariada del ISE-ICA por cuadrante

\begin{tabular}{l|c|c|c}
\hline CUADRANTE & CANTIDAD DE RADIOS & $\begin{array}{c}\text { CANTIDAD DE } \\
\text { POBLACIÓN }\end{array}$ & \% POBLACIÓN \\
\hline I & 363 & 206.511 & 38,91 \\
II & 83 & 55.759 & 10,50 \\
III & 168 & 157.498 & 29,67 \\
IV & 125 & 110.988 & 20,91 \\
\hline TOTAL & 739 & 530.756 & 100,00 \\
\hline
\end{tabular}

El cuadrante I (Figura 4) abarca casi el $40 \%$ de población y la mitad de los radios censales del área de estudio. Su distribución espacial se asemeja al ICA. Le sigue a diez puntos porcentuales de distancia el cuadrante III (Figura 5), que contiene los radios del sector periférico. En esta autocorrelación espacial bivariada los cuadran- 
tes II y, particularmente, el IV (Figuras 6 y 7 ) tienen menor relevancia en el conteo porcentual de población. El primero no posee un patrón definido; no obstante, los radios del norte y sur de la periferia que se encuentran en esta parte corresponden a zonas de crecimiento poblacional hacia donde se dirigen sectores medios en busca de espacios verdes, favorecidos por los bajos costos de los lotes, a pesar de que todavía no cuentan con la totalidad de los servicios. El cuadrante IV distingue una estructura más homogénea, especialmente en el centro y sur de la ciudad, área que comprende un $20 \%$ del total de los habitantes.

Por lo tanto, casi el 70\% de los habitantes de la localidad de Mar del Plata se encuentra en una situación polarizada de acuerdo con el procedimiento utilizado, dado que están repartidos entre el Cuadrante I (radios censales con alto nivel socioeconómico y vecinos con alta calidad ambiental) y el Cuadrante III (radios censales con bajo nivel socioeconómico y vecinos con baja calidad ambiental). El resto se reparte entre el Cuadrante II (radios censales con alto nivel socioeconómico y radios vecinos con baja calidad ambiental) y el Cuadrante IV (radios censales con alto índice de calidad ambiental y vecinos con bajo índice socioeconómico).

\section{FIGURA 4 | Autocorrelación espacial del ISE con el ICA. Cuadrante I}

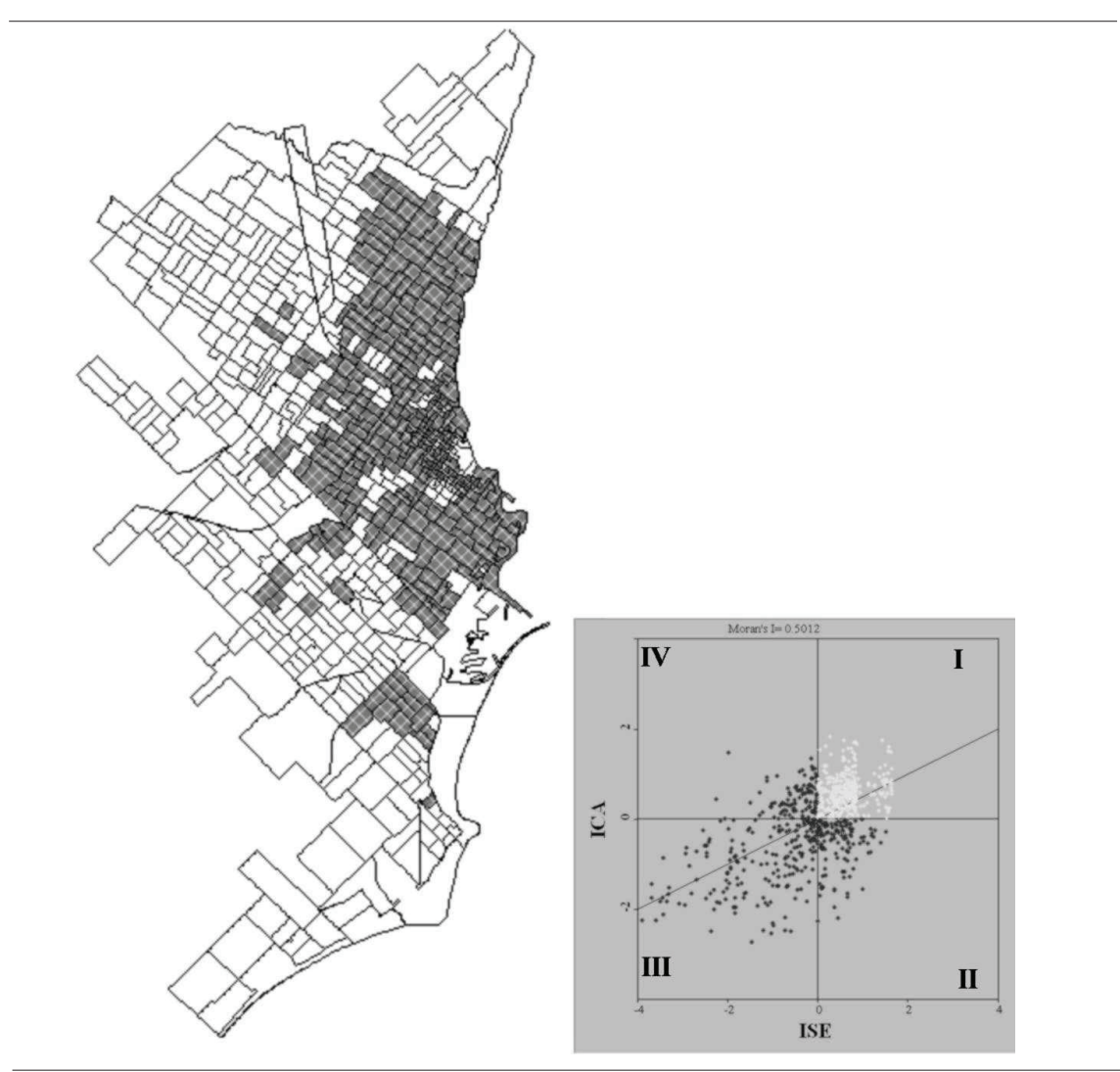

FUeNTE ELABoración Propia CON BASE EN EL PRograma BeOda. 
FIGURA 5 | Autocorrelación espacial ISE con el ICA. Cuadrante III

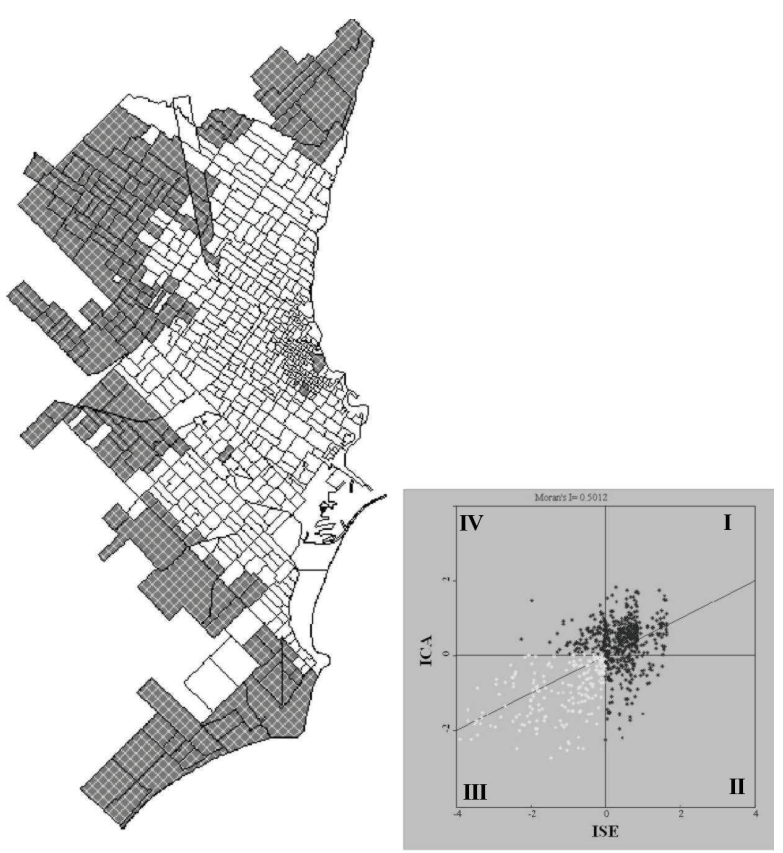

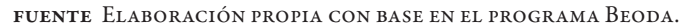

FIGURA 6 | Autocorrelación espacial ISE con el ICA. Cuadrante II

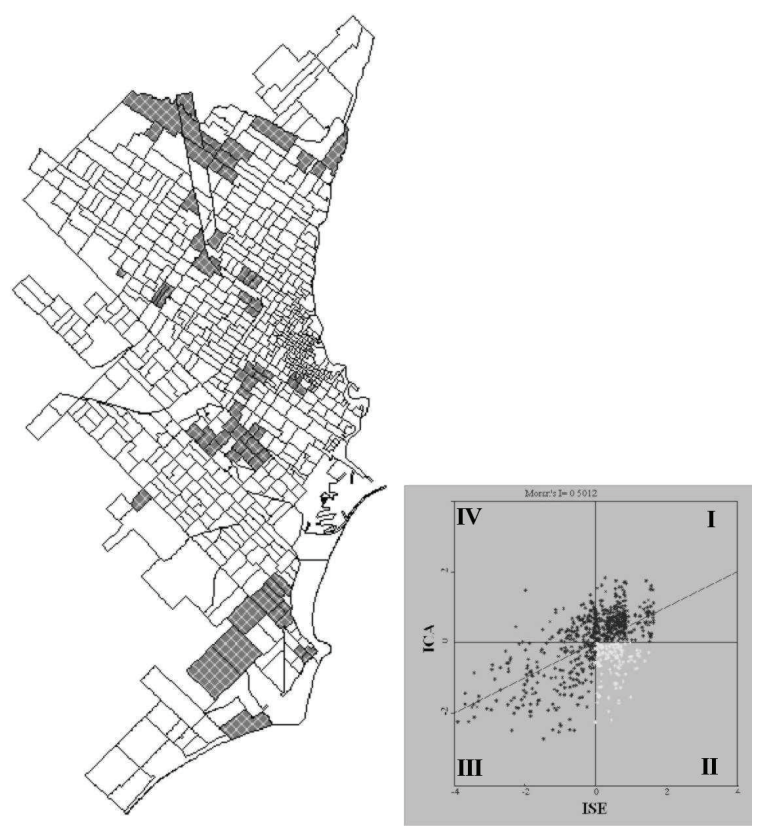

FUente Elaboración propia CON base en EL PRograma Beoda. 
FIGURA 7 | Autocorrelación espacial ISE con el ICA. Cuadrante IV

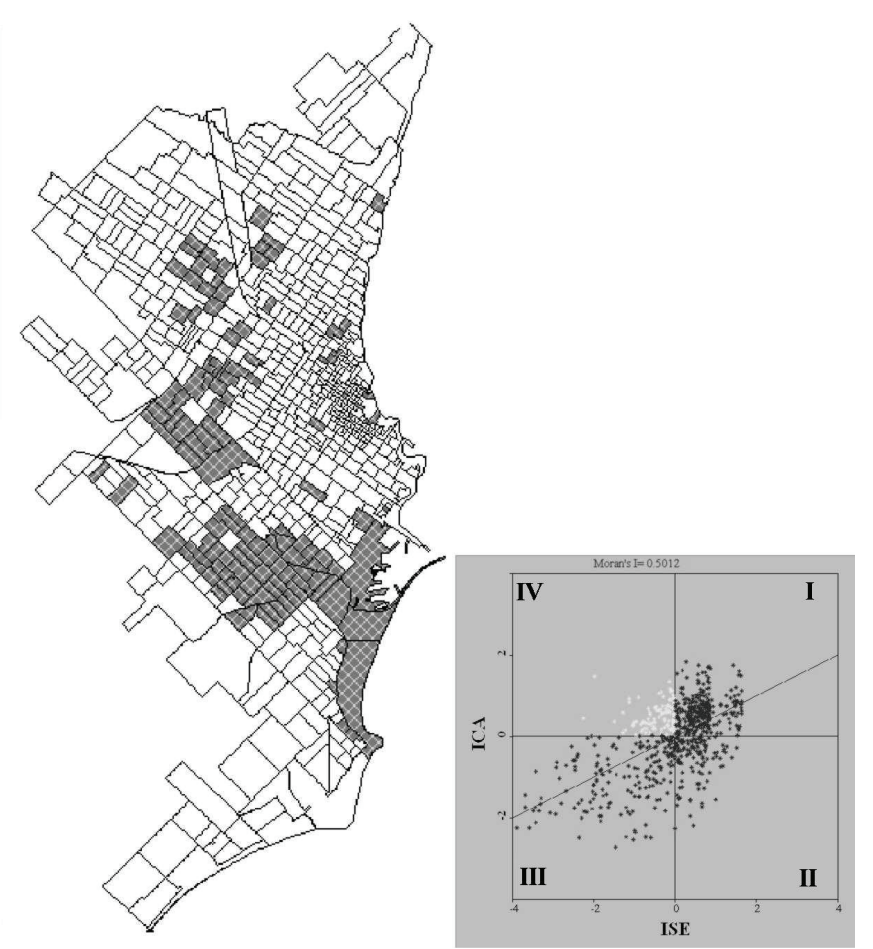

FUENTE ELABORACIÓN PROPIA CON BASE EN EL PROGRAMa GEODA.

\section{Mapa del análisis cluster}

La integración de todas las variables que se encuentran en ambos índices permite realizar un análisis cluster, cuya representación cartográfica con cinco agrupamientos permite distinguir una configuración muy fragmentada (Figura 8), tal como se reconoció con la autocorrelación espacial. Así, se distinguen dos núcleos de color negro (clase 5) compuestos por 103 radios censales, que demuestran ser zonas con un muy buen nivel socioeconómico y ambiental. A su alrededor están 395 radios de color gris claro (clase 2), que actúan como un área de transición hacia la periferia, en la que están representados 132 radios de color blanco (clase 1), con valores bajos en la mayoría de las variables consideradas. Con menor cantidad de unidades espaciales se encuentran 94 radios de color gris oscuro (clase 4), que se asemejan en la distribución espacial al Cuadrante IV de la autocorrelación espacial; es decir, un alto ISE y un bajo ICA. Por último se observan solo 15 radios de color negro con texturas blancas (clase 3), correspondientes a zonas céntricas de la ciudad que presentan signos de deterioro en algunas variables consideradas. 


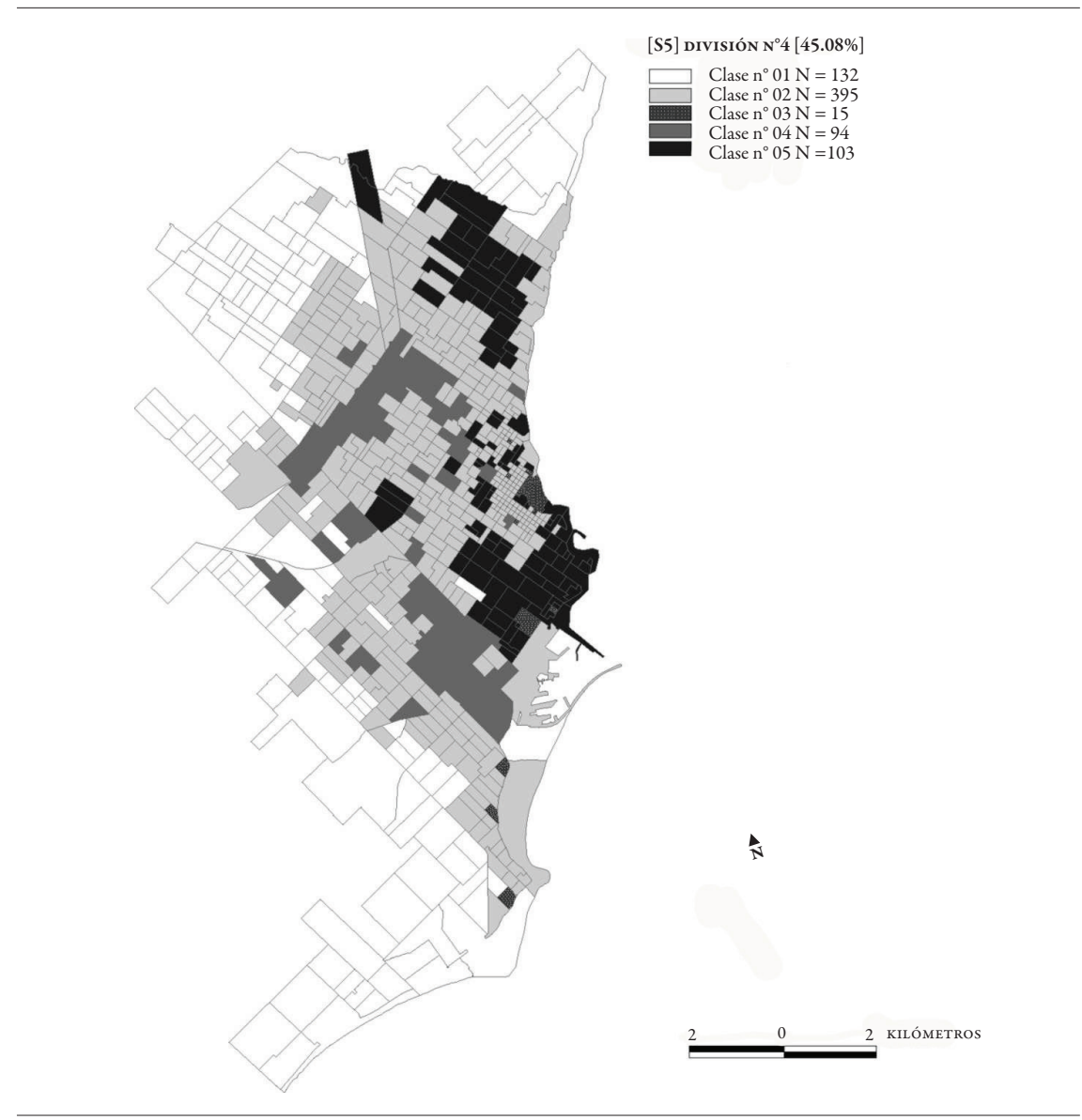

fuente Elaboración propia con base en el Programa Philcarto 5.xx.

\section{Consideraciones finales}

El trabajo buscó aportar al vínculo entre calidad ambiental y las características socioeconómicas de la población desde una visión mayoritariamente empírica. Para ello se construyeron dos índices, uno de índole socioeconómica y otro sobre la calidad ambiental de la ciudad de Mar del Plata (Argentina).

Para su confección y posterior estudio se recurrió a las herramientas desarrolladas en los últimos tiempos en el campo de la información espacial: Sistemas de Información Geográfica (SIG) y programas para el Análisis Exploratorio de Datos Espaciales (ESDA), que permiten mensurar, localizar y estudiar en el territorio las distintas configuraciones resultantes del proceso de fragmentación que se viene registrando 
en los últimos tiempos. El grado de detalle es el máximo posible: el radio censal, compuesto por, aproximadamente, trescientas viviendas. $\mathrm{Al}$ abordar la distribución de los índices desde este tipo de unidad espacial, se logra constatar la fragmentación espacial al interior de la ciudad, un reflejo de la desigualdad en la estructura social que permite reflexionar acerca del papel condicionante del espacio en la evaluación de las condiciones de vida de la población en varias de sus dimensiones.

Los resultados vertidos muestran que las dimensiones ambiental y socioeconómica están interrelacionadas. De esta manera, la capacidad que poseen algunas personas para acceder a una vivienda digna, a un servicio de salud apropiado y a una educación de calidad, también se traslada al componente ambiental. Esto se debe a que el ambiente, en la actualidad, es considerado de manera funcional al contexto socioeconómico prevaleciente y, por lo tanto, sujeto a las directrices del modelo imperante, cuyos rasgos básicos determinan la disposición territorial en el interior de las ciudades. Consecuentemente, se podría decir que el ambiente está "privatizado"; en otras palabras, la disponibilidad de recursos económicos permite acceder o crear mejores condiciones ambientales, a pesar del carácter público que le es inherente. Por lo tanto, las problemáticas ambientales quedan acotadas a aquellos sectores periféricos de la ciudad donde residen las personas de menores recursos.

En definitiva, en cualquiera de los estudios realizados (índices, autocorrelación espacial y análisis cluster) existe un deterioro progresivo en las condiciones de vida de la población en cualquiera de las dimensiones consideradas, en un recorrido desde el centro hacia la periferia de la localidad. Por lo tanto, hacia los sectores de menos recursos no solo hay que implementar políticas que incrementen su situación económica, sino también prevenciones que mejoren el marco ambiental en que habitan. Vivir en un ambiente sano, esto es, sin inundaciones, con abundantes espacios verdes, alejados de focos de contaminación, etcétera, forma parte de los factores esenciales que condicionan el bienestar, el cual, a su vez, no tiene que ser mensurado exclusivamente en términos económicos. IEURE

\section{Referencias}

Alvarado Rosas, C., Medrano \& Lozano, J. (2008). Diferenciación socio-residencial en el área urbana de la ciudad de Cuernavaca, Morelos. Investigaciones Geográficas, 66, 135-152. Recuperado de http://www.revistas.unam.mx/index.php/rig/article/view/17986

Anselin, L. (1993, December 1-5). The Moran Scatterplot as an ESDA tool to asses instability in local association. Documento preparado para su presentación en GISDATA Specialist Meeting on GIS and Spatial Analysis, Ámsterdam, Países Bajos.

Borsdorf, A. (2003). Hacia la ciudad fragmentada. Tempranas estructuras segregadas en la ciudad latinoamericana. Scripta Nova. Revista electrónica de geografía y ciencias sociales, 7, 146(122).

Buzai, G. (2003). Mapas sociales urbanos. Buenos Aires: Lugar Editorial.

Buzai, G. \& Baxendale C. (2006). Análisis socioespacial con Sistemas de Información Geográfica. Buenos Aires: Lugar Editorial / Grupo de Ecología del Paisaje y Medio Ambiente (GEPAMA). 
Celemín, J. \& Velázquez, G. (2011). Estimación de un índice de calidad ambiental para la Ciudad y Provincia de Buenos Aires. Journal of Latin American Geography; 10(1), 71-84. doi: 10.1353/lag.2011.0017

Cepeda, R., Marinelli, C., Gómez Lende S. \& Velázquez G. (2004).Técnicas de análisis multivariado para la determinación de calidad de vida. Memorias del Primer Seminario Argentino de Geografía Cuantitativa, Buenos Aires, Argentina.

Clichevsky, N. (2002). Pobreza y politicas urbano-ambientales en Argentina. CEPAL Serie Medio Ambiente y Desarrollo, 49. Santiago: CEPAL, División de Medio Ambiente y Asentamientos Humanos.

De Mattos, C. \& Hidalgo, R. (Eds.). (2007). Santiago de Chile: Movilidad espacial y reconfiguración metropolitana. Santiago: Colección Eure Libros / Geolibros.

Di Pace, M., Federovisky, S., Hardoy, J. \& Mazzucchelli, S. (1992). Medio ambiente urbano en la Argentina. Colección Los fundamentos de las ciencias del hombre. Buenos Aires: Centro Editor de América Latina (CEAL).

Escobedo, F. J., Nowak, D. J., Wagner, J., De la Maza, C. L, Rodríguez, M., Crane, D. \& Hernández, J. (2006). The socioeconomics and management of Santiago de Chile's public urban forests. Urban Forestry and Urban Greening, 4, 105-114. Disponible online en www.sciencedirect.com

Gómez Lende, S. (2005). La fragmentación de la calidad de vida en el conurbano bonaerense (19912001). En G. Velázquez \& S. Gómez Lende (autores/comp.), Desigualdad y calidad de vida en la Argentina (1991-2001). Aportes empiricos y metodológicos (pp. 241-280). Tandil (Provincia de Buenos Aires): Red de Editoriales Universitarias Nacionales (REUN).

González Arellano, S. \& Villeneuve, P. (2007). Transformaciones en el espacio sociorresidencial de Monterrey, 1990-2000. Estudios Demográficos y Urbanos, 22 (1 64), 143-178.

Goodchild, M. (1988). Spatial autocorrelation. Reino Unido: Geo Books.

Groisman, F. \& Suárez, A. L. (2006). Segregación residencial en la Ciudad de Buenos Aires. Revista de Población de Buenos Aires, 3(4), 27-37.

Janoschka, M. (2002). El nuevo modelo de la ciudad latinoamericana: fragmentación y privatización. EURE, 28(85), 11-29. doi: 10.4067/S0250-71612002008500002

Jensen, R., Gatrell, J., Boulton, J. \& Harper, B. (2004). Using remote sensing and geographic information systems to study urban quality of life and urban forest amenities. Ecology and Society, 9(5). Disponible en http://www.ecologyandsociety.org/vol9/iss5/art5/

Kaztman, R. \& Retamoso, A. (2007). Efectos de la segregación urbana sobre la educación en Montevideo. Revista de la CEPAL., 9(1), 133-152. Disponible en http://www.eclac.org /publicaciones/xml/3/28263/LCG2333eKaztman.pdf

Linares, S. (2008). Viejas técnicas, nuevas tecnologías: El aporte de los SIGs al análisis de la segregación socioespacial urbana. Geodemos (Buenos Aires) (en prensa).

Linares, S. (2010). Aplicación de Sistemas de Información Geográfica al estudio de la segregación socioespacial urbana: el caso de la ciudad de Tandil. En G. Buzai (Ed.), Geografía y Sistemas de Información Geográfica. Aspectos conceptuales y aplicaciones (pp. 329-392). Luján: Grupo de Estudios sobre Geografía y Análisis Espacial con Sistemas de Información Geográfica (GESIG), Universidad Nacional de Luján. 
Lucero, P. \& Celemín, J. (2008). La calidad de vida de la población en la determinación de la calidad territorial. Un estudio de autocorrelación espacial aplicado a la ciudad de Mar del Plata. GeoFocus. Revista Internacional de Ciencia y Tecnología de la Información Geográfica, 8, 94114. Disponible en http://geofocus.rediris.es/2008/Articulo5_2008.pdf

Martori, J.; Hoberg, K. \& Surinach, J. (2006). Población inmigrante y espacio urbano: Indicadores de segregación y pautas de localización. EURE, 32(97), 49-62. doi: 10.4067/ S0250-71612006000300004

Mennis, J. (2006). Socioeconomic-vegetation relationships in urban, residential land: The case of Denver, Colorado. Photogrammetric Engineering \& Remote Sensing, 72, 911-921. Disponible en http://astro.temple.edu/ jmennis/pubs/mennis_pers06.pdf

Meyer, K. \& Bähr, J. (2001). Condominios in Greater Santiago de Chile and their impact on the urban structure. Die Erde, 132(3), 293-321.

Openshaw, S. (1984). The modifiable areal unit problem. Norwich: Geo Books.

Pedlowski, M. A., Carneiro Dasilva, V. A., Adell, J. J. C., \& Heynen, N. C.. (2002). Urban forest and environmental inequality in Campos dos Goytacazes, Rio de Janeiro, Brazil. Urban Ecosystems, 6(1-2), 9-20. doi: 10.1023/A:1025910528583

Sabatini, F.; Cáceres, G. \& Cerda, J. (2001). Segregación residencial en las principales ciudades chilenas: Tendencias de las tres últimas décadas y posibles cursos de acción. EURE, 28(82), 21-42. doi: 10.4067/S0250-71612001008200002

Sachs-Jeantet, C. (1995). Ciudad y gestión de las transformaciones sociales. Unesco, Documentos de debate, 2, 1-24.

Santana Rodríguez, L., Escobar Jaramillo, L. \& Capote, P. 2010. Estimación de un índice de calidad ambiental urbano, a partir de imágenes de satélite. Norte Grande, 45, 77-95. doi: 10.4067 /S0718-34022010000100006

Stathopoulou, M. (2009). Quality of life in metropolitan Athens using satellite and census data: Comparison between 1991 and 2001. Documento presentado en Second International Conference on Countermeasures to Urban Heat Islands, Berkeley, California.

Uribe Ortega, G. (1998). Geografía y sociedad. Exploraciones en compromisos y propuestas actuales. México: Centro de Investigaciones Científicas.

Velázquez, G. (2008). Viejas inequidades, nuevos dimensionamientos: "centros" y "periferias" como elementos para el análisis geográfico del bienestar de la población argentina luego del censo de 2001. En G. Velázquez \& N. Formiga (Coords.), Calidad de vida, diferenciación socioespacial y condiciones sociodemográficas. Aportes para su estudio en la Argentina (pp. 23-47). Bahía Blanca (Provincia de Buenos Aires): Editorial de la Universidad Nacional del Sur. 